\title{
REDISTRIBUCIÓN DE PLANTA Y PROGRAMACIÓN DE LA PRODUCCIÓN: UN ENFOQUE INTEGRADO
}

\author{
Daniel Jaramilloํㅗ Juliana Uriarte ${ }^{1}$, Luis Felipe Cardona ${ }^{2}$
}

\author{
${ }^{1}$ Estudiante de Ingeniería Industrial \\ Universidad ICESI, Cali, Colombia \\ ${ }^{2}$ Estudiante de Doctorado de Ingeniería Industrial, KY, EE.UU. \\ Correo electrónico: luis.cardonaolarte@louisville.edu
}

Recibido: 5 de mayo del 2014. Aprobado: 20 de julio del 2014.

Cómo citar este artículo: D. Jaramillo, J. Uriarte y L. F. Cardona, "Redistribución de planta y programación de la producción: un enfoque integrado". Ingeniería Solidaria, vol. 10, n. ${ }^{\circ}$ 17, pp. 71-81, en.-dic., 2014. doi: http://dx.doi.org/10.16925/in.v9i17.807

Resumen. La preocupación principal de las empresas al realizar un proyecto de redistribución de planta es la interrupción de las operaciones de manufactura mientras se trasladan los departamentos. Este artículo presenta un modelo de optimización para programar el traslado de los departamentos y la producción, de tal manera que se garantice el cumplimiento de la demanda durante la ejecución del proyecto de redistribución de planta. Con este propósito, se planteó un modelo de programación lineal entera mixta (PEM) que minimiza los costos de traslado de los departamentos, asegura el cumplimiento de la demanda y limita la duración del proyecto a un máximo establecido. Los resultados muestran que cuando la utilización promedio de la maquinaria de la planta es mayor al $60 \%$, el proyecto de redistribución y la programación de la producción son interdependientes, por lo tanto es necesario utilizar un enfoque integrado como el propuesto en este artículo. Este se deriva del proyecto de investigación "Optimización de procesos de redistribución de planta en manufactura flexible” desarrollado en la Universidad IcEsi a lo largo del periodo 2013-2014.

Palabras clave: distribución de planta, redistribución de planta, optimización, programación de proyectos.

\section{Production Plant Redistribution and SCHEDUling: AN Integrated ApProACH}

Abstract. The main concern of companies when carrying out a plant redistribution project is the interruption of manufacturing operations while departments are transferred. This article presents an optimization model for scheduling the transference of departments and production, in order to guarantee fulfillment of demand during a plant redistribution project execution. For this purpose, a mixed complete linear programming model (PEM from its Spanish acronym) was applied that minimizes the cost of transferring departments, ensures demand fulfillment and limits the duration of the project to an established maximum. The results show that, when average plant machinery use is greater than $60 \%$, the redistribution project and production scheduling are interdependent, thus the need for an integrated approach such as the one proposed in this article. This is derived from the research project "Optimization of Plant Redistribution Processes in Flexible Manufacturing" ("Optimización de procesos de redistribución de planta en manufactura flexible") carried out at the Universidad ICESI in 2013-2014.

Keywords: plant distribution, plant redistribution, optimization, project scheduling.

\section{REDISTRIBUIÇÃo DE FÁBRICA E PROGRAMAÇÃo DA PRODUÇÃO: UM ENFOQUE INTEGRADO}

Resumo. A preocupação principal das empresas ao realizarem um projeto de redistribuição de fábrica é a interrupção das operações de manufatura enquanto se deslocam os departamentos. Este artigo apresenta um modelo de otimização para programar a mudança dos departamentos e a produção, de maneira que se garanta o cumprimento da demanda durante a execução do projeto de redistribuição de fábrica. Com esse propósito, propôs-se um modelo de programação linear inteira mista (PEM) que minimiza os custos de deslocamento dos departamentos, garante o cumprimento da demanda e limita a duração do projeto a um máximo estabelecido. Os resultados mostram que, quando a utilização média do maquinário da fábrica é maior a $60 \%$, o projeto de redistribuição e a programação da produção são interdependentes; portanto, é necessário utilizar um enfoque integrado como o proposto neste artigo. Este se deriva do projeto de pesquisa "Otimização de processos de redistribuição de fábrica em manufatura flexível", desenvolvido na Universidade ICESI em 2013-2014.

Palavras-chave: distribuição de fábrica, redistribuição de fábrica, otimização, programação de projetos. 


\section{Introducción}

En distribución de planta, la mayoría de la literatura se ha concentrado en el diseño de una nueva instalación. En los últimos años, se ha encontrado que es más común que una empresa se enfrente a un proyecto de redistribución que a uno de distribución de planta [1]. La situación se debe a la alta variabilidad del entorno al que se enfrentan las empresas hoy en día [2].

El problema de redistribución de planta consiste en pasar de una distribución existente a una nueva distribución minimizando tanto los costos de manejo de materiales como los costos de la redistribución, teniendo en cuenta la adición de nuevos departamentos o modificaciones en las áreas o posiciones de los departamentos existentes [2]. Muy pocas investigaciones se han hecho acerca de la programación óptima de las actividades para implementar una redistribución de planta, aun cuando la implementación es crítica para lograr el éxito del proyecto de redistribución [3]. Las actividades del proyecto son tanto el trabajo de preparación como los movimientos físicos de los departamentos necesarios para lograr la nueva distribución de planta [3]. Driscoll y Sawyer [4] desarrollaron un procedimiento heurístico de programación de actividades para un cambio gradual, el cual identifica las series de movimientos de departamentos por realizar durante un intervalo de tiempo para pasar de una distribución de planta a otra. Lacksonen y Hung [3] desarrollaron el problema de programación de actividades en un proyecto de redistribución de planta, el cual es modelado como un problema de programación entera mixta con dos objetivos.

Dentro de los estudios anteriormente desarrollados sobre programación de las actividades para implementar una distribución de planta, solo se ha tenido en cuenta la minimización de los costos de la redistribución y del tiempo del proyecto [5]; sin embargo, no se ha tenido en cuenta que la principal preocupación de las empresas al realizar un proyecto de redistribución de planta es no poder cumplir con la demanda debido a las interrupciones de la producción [6].

En este artículo, se presenta un modelo de programación lineal entera mixta el cual integra la programación de las actividades de un proyecto de redistribución de planta con la planeación de producción para garantizar el cumplimiento de la demanda durante el desarrollo del proyecto. Se encontró que cuando la utilización de las máquinas de la planta es alta, es necesario tener en cuenta la planeación de la producción durante el proyecto de redistribución para que la planta pueda cum- plir con la demanda; por lo tanto, en estas ocasiones el modelo planteado en este artículo es el más adecuado.

La sección 2 describe el problema de planeación de proyectos de redistribución de planta y muestra los estudios realizados hasta ahora sobre el tema. La sección 3 enseña el modelo de optimización planteado en este artículo y lo explica a través de un ejemplo. La sección 4 plantea los resultados obtenidos. Finalmente, la sección 5 presenta las conclusiones y futuras investigaciones.

\section{Antecedentes}

Rosenblatt [7] fue el primero en considerar la dinámica del problema de distribución en un horizonte de tiempo, anticipando los cambios en la distribución de la planta en respuesta a demandas pronosticadas. Seguidamente, Batta [8], Urban [9] y [10] y Balakrishnan [11] contribuyeron a mejorar el procedimiento propuesto por Rosenblatt [7] al establecer límites, tanto superiores como inferiores, para la inversión total del proyecto y proponiendo procedimientos de solución que encuentran mejores resultados para los objetivos establecidos.

Para Liu [12], el problema de distribución de planta dinámico consiste en asignar los departamentos a posiciones predefinidas durante un horizonte de tiempo de tal manera que los costos del manejo de materiales y rediseños sean mínimos. Montreuil y Venkatadri [13] presentaron la primera formulación de un problema de distribución de planta dinámico usando departamentos con áreas y formas desiguales. Este modelo fue mejorado por Montreuil y Laforge [14] que incluyeron escenarios probabilísticos en el horizonte de tiempo. Lacksonen y Emory [15] sugieren incorporar los cambios futuros que pueden ser anticipados en la distribución de planta como un acercamiento para generar diseños flexibles. Después, Lacksonen [16] propuso un modelo heurístico de dos etapas para resolver el problema de distribución de planta dinámico mediante departamentos con áreas diferentes. Balakrishnan y Cheng [17] resaltan la importancia de utilizar métodos de simulación y heurísticos en problemas de distribución de planta dinámicos que permiten considerar características más realistas en el problema. Posteriormente, Maziani, Abedzadeh y Mohebali [18] representan la estructura de la planta mediante bandas flexibles y utilizan algoritmos genéticos para resolver el problema.

La tendencia más reciente de los modelos de distribución de planta dinámicos es generar distribuciones 
de planta flexibles que se puedan adaptar fácilmente a los cambios del entorno [19], y es en este contexto en el que la redistribución de planta se convierte en la estrategia de las organizaciones para mantener la eficiencia de sus distribuciones [20], [21].

El problema de redistribución de planta es un caso especial del problema de distribución de planta dinámico, en el que el primer periodo para considerar es el presente, se cuenta con una distribución inicial para este primer periodo y sólo se consideran dos periodos de tiempo en el análisis [2]. Este problema comparte muchas características con el problema de distribución de planta dinámico, pero es necesario realizar ciertas consideraciones adicionales [2] como las variaciones de las áreas de los departamentos, la existencia o no de maquinaria fija en los departamentos y la entrada y salida de departamentos en la distribución. Hicks y Lowan [22] fueron los primeros en tener en cuenta los costos de reubicar los departamentos en un problema de distribución de planta. Kulturel-Konak, Smith y Norman [2] presentan un enfoque bi-objetivo para resolver el problema de redistribución con departamentos expandibles o fijos en el que se minimizan los costos de manejo de materiales y los costos de reorganización. Ferrari, Pareschi, Persona y Regattieri [23] presentaron un software integrado con formatos CAD (Computer-Aided Design) para implementar proyectos de redistribución, en el cual se consideran tanto los costos de manejo de materiales como las relaciones cualitativas entre los departamentos. Adicionalmente, Savsar [24] y Discroll y Sawyer [4] presentaron métodos sistemáticos y algoritmos basados en simulación para resolver problemas de distribución de planta flexibles, en los que se tiene entre los objetivos minimizar los costos esperados de redistribución. Han, Bae y Jeong [21] presentaron un método para comparar la eficiencia de diferentes alternativas de diseños para la planta a partir de modelos de simulación. A pesar de los avances en el campo, el tema de redistribución de planta sigue siendo poco estudiado y necesita más investigación [25] y [26].

Meng, Heragu y Zijm [27] proponen una metodología de 4 fases para la realización de proyectos de redistribución de planta. La fase 1 consiste en generar diseños candidatos; la fase 2 se trata de evaluar el desempeño de los diseños propuestos en la fase 1 . La fase 3 consiste en seleccionar el diseño que se va a utilizar. Por último, la fase 4 consiste en refinar el diseño seleccionado a partir de un análisis interdisciplinar. Posteriormente, Rivera, Cardona, Vásquez y Rodríguez [6] propusieron incluir la fase 5: realizar el plan de implementación del proyec- to de redistribución. Hasta el momento, la mayoría de las investigaciones se han concentrado en la fase 1. Muy pocas se han enfocado en la fase 5 .

Los primeros en trabajar en la fase 5 fueron Driscoll y Sawyer [4], que desarrollaron un programa de simulación que facilita la programación de las actividades para pasar de una distribución de planta antigua a una nueva. Lacksonen y Hung [3] desarrollaron un modelo matemático de programación lineal que permite realizar la programación de los movimientos de los departamentos en un proyecto de redistribución de planta y minimiza los costos del traslado de los departamentos. Posteriormente, Rivera et al. [6] encontraron que la principal preocupación de las empresas a la hora de realizar un proyecto de redistribución de planta es la interrupción de los procesos de manufactura y en consecuencia el incumplimiento en los pedidos a los clientes. Es por esto que en este artículo se integra el modelo de programación de proyectos de redistribución de planta con la programación de producción, de tal manera que se garantiza el cumplimiento de la demanda durante el desarrollo del proyecto de redistribución de planta.

\section{Modelo matemático}

Lacksonen y Hung [3] presentan un modelo para programar el traslado de los departamentos en un proyecto de redistribución de planta que minimiza simultáneamente los costos de traslados de los departamentos y la duración total del proyecto. En esta sección se presenta un modelo de programación lineal entera mixta que integra la planeación de producción al modelo propuesto por Lacksonen y Hung [3], de tal manera que se garantice el cumplimiento de la demanda durante el proyecto de redistribución de planta.

Para asegurar el cumplimiento de la demanda, el modelo tiene en cuenta la capacidad de operación de cada departamento y organiza el proyecto de tal manera que se produzca inventario antes de trasladar los departamentos para abastecer la demanda mientras los departamentos se encuentran fuera de operación. El objetivo del modelo es minimizar los costos de traslado de los departamentos limitando la duración del proyecto a un tiempo máximo.

Para programar el traslado de los departamentos el modelo utiliza el grafo de precedencias propuesto por Lacksonen y Hung [3], donde las precedencias se obtienen sobreponiendo la distribución deseada sobre la distribución actual: "Si un departamento en la dis- 
tribución inicial esta debajo de un departamento en la distribución final entonces el movimiento del departamento debe preceder el movimiento del departamento, siempre que ". Adicionalmente se tiene en cuenta que no todos los departamentos cambian de posición durante el proyecto, por lo tanto, sólo existirán precedencias entre los departamentos que cambien de posición en la distribución.

Para coordinar los traslados de los departamentos, se utiliza un espacio de ubicación temporal a donde los departamentos van para liberar espacio en el interior de la planta y permitir el traslado de otros departamentos. En el modelo se consideró que hay departamentos con maquinaria liviana y bajos costos de ajuste que pueden operar mientras estén en ubicaciones temporales, así como hay departamentos de maquinaria pesada que dados sus costos de instalación, no producen mientras se encuentren en ubicaciones temporales.

A continuación, se explica detalladamente los conjuntos, parámetros, variables, función objetivo y restricciones del modelo.

- Conjuntos: los departamentos (subíndice: i), los productos (subíndice: $j$ ), los días (subíndice: $d$ ) y los turnos (subíndice: $s$ ), en los que cada día esta compuesto por varios turnos.

- Parámetros: las demandas diarias de cada producto $\left(D_{j i}\right)$; los turnos correspondientes a cada día $\left(D P E R_{s d}\right)$; si un departamento se traslada o no $\left(\right.$ DEPTMOV $\left._{i}\right)$; el tiempo de producción de cada producto en cada departamento $\left(P T_{j i}\right)$; la ruta de producción $\left(P R T_{i}\right)$; la duración de cada turno (PTA); los tiempos de preparación $\left(P R T_{i}\right)$, de transporte $\left(T T_{i}\right)$ y de ajuste $\left(T A_{i}\right)$ de cada departamento; la matriz de precedencias de los traslados de los departamentos y el inventario inicial de cada producto (INVINI ${ }_{j}$ ). El límite máximo que puede durar el proyecto $\left(\right.$ TMAXX $\left._{i}\right)$, el número total de turnos (MPER), un parámetro auxiliar $(M)$ que representa un número muy grande, el costo de trasladar cada departamento $\left(M O V C_{i}\right)$, el área de cada departamento y el área máxima que se puede utilizar como ubicaciones temporales de los departamentos (TURNAREA).

- Variables: la producción de cada producto en cada turno $\left(P N_{j s}\right)$; el inventario al final de cada día de cada producto $\left(I N V_{j d}\right)$; una variable auxiliar para asegurar la capacidad de producción de los departamentos $\left(P N M_{j s i}\right)$; el turno en que cada departamento inicia $\left(\right.$ TINI $\left._{i s}\right)$ y termina $\left(\right.$ TFIN $\left._{i s}\right)$; el traslado a su posición final, turno de inicio (DTINI ${ }_{i s}$ y turno de finalización $\left(D_{T F I N}{ }_{i s}\right)$ del movimiento del departamento a su posición temporal; la duración total del proyecto $(T M A X)$; si un departamento va a una posición temporal $\left(Y_{i}\right)$; si un departamento está fuera de funcionamiento o no en cada turno $\left(S D_{i s}\right)$; y si un departamento está en una posición temporal o no en cada turno $\left(T U R N_{i s}\right)$.

- Función objetivo: minimizar los costos del proyecto. Los cuales se pueden resumir en la suma de los costos de los movimientos a posiciones temporales, ya que todos los demás costos son fijos (1).

- Restricciones: la (2) y la (3) garantizan un sólo inicio y un solo final respectivamente, para los movimientos de departamentos a su posición final. Las restricciones (4) y (5) se encargan de garantizar un sólo inicio y un sólo final respectivamente para movimientos de departamentos a una posición temporal. Las restricciones (6) y (7) sirven para definir el momento en el que finaliza el movimiento a la posición final y temporal respectivamente. La restricción (8) limita la duración del proyecto a un tiempo máximo dado. La restricción (9) define el inventario del día uno, mientras que la restricción (10) define este para todos los demás días. La restricción (11) limita la producción diaria según la capacidad de las máquinas, mientas que la restricción (12) se encarga de limitarla según el tiempo disponible. Las restricciones (13) y (14) se encargan de definir la duración de los movimientos. Las restricciones (15) y (16) definen las precedencias si no va al temporal y si va respectivamente, la restricción (17) sirve para definir que el tiempo de finalizar el movimiento final debe ser mayor al tiempo final de ir al temporal para cada departamento. Las restricciones (18), (19), (20) y (21) limitan el área del espacio temporal a lo largo del proyecto. La restricción (22) limita la duración del proyecto.

El modelo de optimización completo se presenta a continuación.

\section{Conjuntos:}

Departamentos $i$

Turnos $s$

Productos $j$

Días $d$ 


\section{Parámetros:}

$D_{j i}:$ demanda diaria de cada producto.

$D P E R_{s d}:$ turnos correspondientes a cada día.

DEPTMOV: $_{i}$ si el departamento se mueve.

$P T_{j i}$ : tiempo de producción de cada producto en cada departamento.

$P R_{j i}:$ ruta de producción.

PTA: cantidad de tiempo de un turno.

$P R T_{i}$ : tiempo de preparación de cada departamento.

$T T_{i}$ : tiempo de transporte de cada departamento.

$T A_{i}$ : tiempo de ajuste de cada departamento.

$P_{i l}:$ indica si el movimiento de un departamento es predecesor de otro.

INVINI: inventario inicial de cada producto.

TMAXX: tiempo máximo de duración del proyecto.

MPER: número total de turnos.

$M$ : un número grande.

MOVC $_{i}$ : costo de mover cada departamento.

AREAS: $:$ área de cada departamento.

TURNAREA: área del espacio temporal.

\section{Variables:}

$P N_{j s}$ : producción del producto en el turno

$I N V_{j d}$ : inventario del producto el día

$P N M_{j s i}:$ producción producto en turno en el departamento .

$\operatorname{TIN}_{i s}$ : turno en el que el departamento inicia el movimiento a su posición final.

$T_{F I N_{i s}}$ : turno en el que el departamento finaliza el movimiento a su posición final.

$D T_{I N I}$ : turno en el que el departamento inicia el movimiento a su posición temporal. turno en el que el departamento finaliza el movimiento a su posición temporal.

$D_{\text {DTFIN }}$ : indica si el departamento va a una posición temporal (binaria).

$Y_{i}$ : Indica si el departamento se encuentra funcionando en el turno (binaria).

duración del proyecto.

$S D_{i s}$ : indica si el departamento se encuentra funcionando en el turno (binaria).

\section{Función objetivo:}

$\operatorname{Min} \mathrm{Z}=\sum_{i} Y_{i} * \mathrm{MOVC}_{i}$

\section{Restricciones:}

$$
\begin{aligned}
& \sum_{s} T_{T N I_{i s}}=\text { DEPTMOV }_{i} \\
& \Sigma_{s} \text { TFIN }_{i s}=\text { DEPTMOV } \\
& \sum_{s} T_{I N I_{i s}}=Y_{i} \\
& \sum_{s} \text { DTFIN }_{i s}=Y_{i} \\
& \sum_{s}\left(\text { TFIN }_{i s} * S\right)=\Sigma_{s}\left(\text { TINI }_{i s} * S\right)+\left(P R T_{i}+T T_{i}+T A_{i}\right) \text { DEPTMOV }_{i} \\
& \sum_{s}\left(D_{T F I N}{ }_{i s} * S\right)=\sum_{s}\left(D T I N I_{i s} * S\right)+\left(P R T_{i}+T T_{i}+T A_{i}\right) Y_{i} \\
& \sum_{s}\left(\operatorname{TFIN}_{i s} * S\right) \leq T M A X \\
& I N V_{j 1}=\Sigma_{s}\left(D P E R_{j 1} * P N_{j 1}\right)+I N V I N I_{j}-D_{j 1} \\
& I N V_{j d}=\sum_{1}^{s}\left(D P E R_{s, d} * P N_{j s}\right)+I N V_{j d-1}-D_{j d} \\
& P N_{j s} \geq P N M A Q_{j s i} \\
& \sum_{j}\left(P N M A Q_{j s i} P T_{j i} P R_{j i}\right) \leq P T A\left(1-S D_{i s}\right) \\
& S D_{i s} \geq \sum_{s-P R T_{i}-T T_{i}-T A_{i}+1}^{s} T F I N_{i l}+\sum_{s-P R T_{i}-T T_{i}-T A_{i}+1}^{s} D T I N I_{i l} \\
& S D_{i s} \geq \sum_{1}^{s} T^{\prime N I I_{i l}}+\sum_{1}^{s} D_{T I N I_{i l}} \forall_{s<-P R T_{i}+T A_{i}-1}
\end{aligned}
$$$$
\forall_{j} \forall_{d \leq 2}(10)
$$$$
\forall_{j} \forall_{s} \forall_{i}(11)
$$$$
\forall_{i} \forall_{s}(12)
$$$$
\forall_{i} \forall_{s \geq P R T_{i}+T T_{i}+T A_{i}}(13)
$$$$
\forall_{i}(14)
$$ 


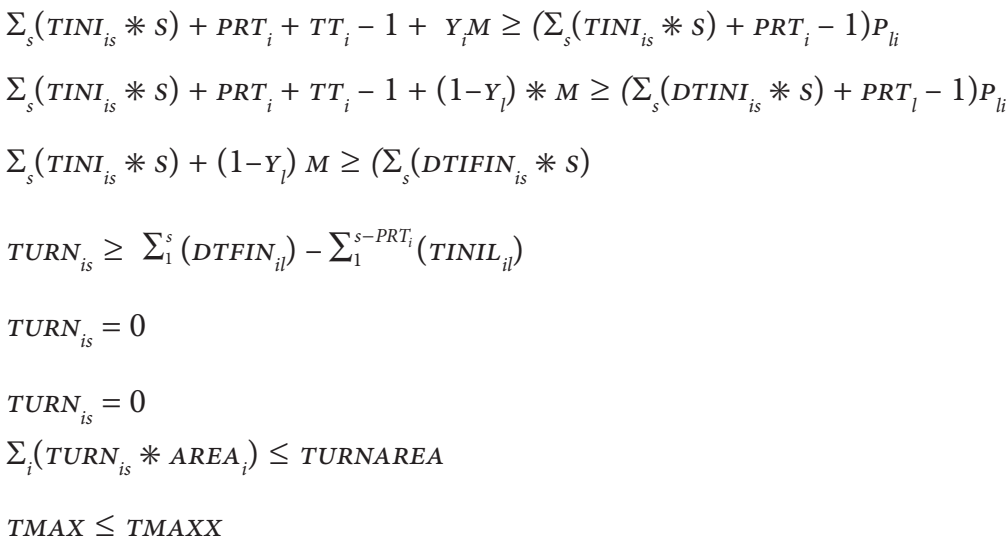

$\sum_{s}\left(T I N I_{i s} * S\right)+P R T_{i}+T T_{i}-1+Y_{i} M \geq\left(\sum_{s}\left(T I N I_{i s} * S\right)+P R T_{i}-1\right) P_{l i}$

$\forall_{i \neq j}(15)$

$\forall_{i \neq j}(16)$

$\forall_{i}(17)$

$\forall_{i} \forall_{M T E R-T A_{i} \leq s \geq P R T_{l+1}}$

$$
\begin{array}{r}
\forall_{i} \forall_{s \leq P R T_{i}}(19) \\
\forall_{i} \forall_{s \geq M P E R-T A_{i}+1}(20) \\
\forall_{s}(21)
\end{array}
$$

Para ilustrar este modelo, se presenta un ejemplo tran en las figuras 1 (a) y (b), respectivamente, donde la cafetería, el espacio vacío y la estatua no representan departamentos productivos.

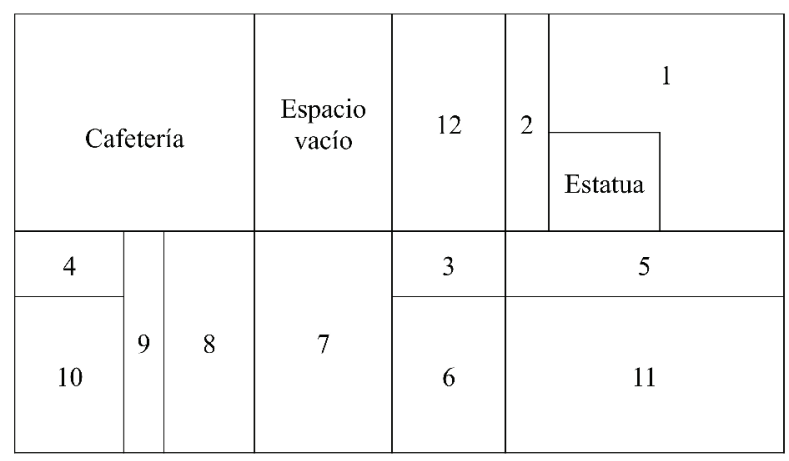

(a)

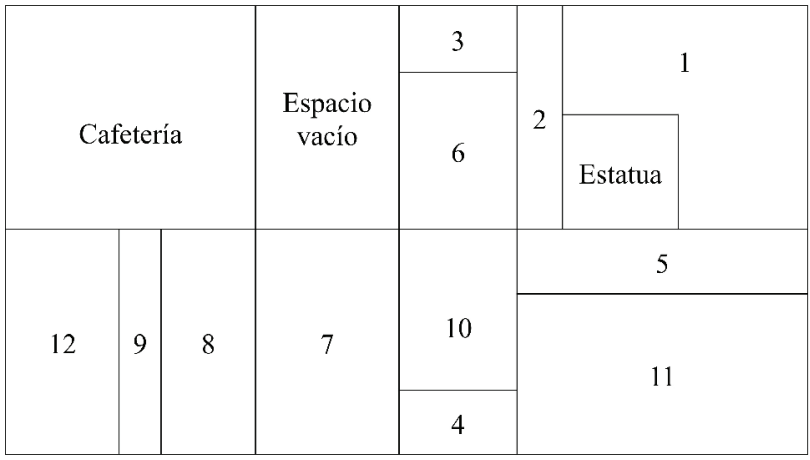

(b)

Figura 1. (a) Distribución inicial de planta. (b) Distribución final de planta Fuente: [3]

La figura 2 muestra el grafo de precedencias de los traslados de los departamentos. Por ejemplo, el departamento 12 es precedencia del departamento 3, de tal manera que se debe mover el departamento 12 primero para que el departamento 3 pueda llegar a su posición final.

Los datos utilizados en el ejemplo son los presentados en las tablas 1-5.

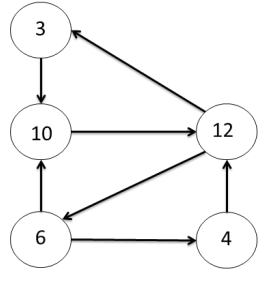

Figura 2. Grafo de precedencias Fuente: elaboración propia 
Tabla 1. Demanda (D)

\begin{tabular}{|l|c|c|c|c|c|c|}
\hline & PRT & TT & TA & MOVC & ÁREAS & DEPTMOV \\
\hline Dept 1 & 2 & 3 & 1 & 8000 & 8000 & 1 \\
\hline Dept 2 & 1 & 2 & 1 & 4800 & 2400 & 1 \\
\hline Dept 3 & 2 & 1 & 1 & 2800 & 2800 & 1 \\
\hline Dept 4 & 1 & 1 & 1 & 2000 & 2000 & 1 \\
\hline Dept 5 & 2 & 2 & 1 & 8400 & 4200 & 1 \\
\hline Dept 6 & 1 & 1 & 1 & 3600 & 3600 & 1 \\
\hline Dept 7 & 2 & 4 & 1 & 8000 & 8000 & 1 \\
\hline Dept 8 & 1 & 2 & 1 & 5200 & 5200 & 1 \\
\hline Dept 9 & 1 & 2 & 1 & 3900 & 3900 & 1 \\
\hline Dept 10 & 2 & 2 & 1 & 22500 & 4400 & 1 \\
\hline Dept 11 & 1 & 5 & 1 & 54000 & 10800 & 1 \\
\hline Dept 12 & 1 & 2 & 1 & 4800 & 4800 & 1 \\
\hline
\end{tabular}

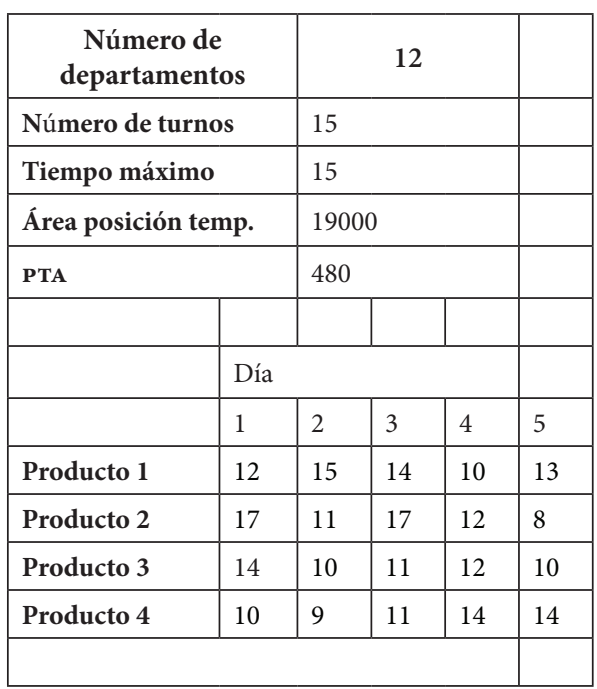

Fuente: elaboración propia

Tabla 2. Precedencias $(\mathrm{P})$

\begin{tabular}{|l|c|c|c|c|c|c|c|c|c|c|c|c|}
\hline & \multicolumn{10}{|c|}{ Departamento } \\
\hline & $\mathbf{1}$ & $\mathbf{2}$ & $\mathbf{3}$ & $\mathbf{4}$ & $\mathbf{5}$ & $\mathbf{6}$ & $\mathbf{7}$ & $\mathbf{8}$ & $\mathbf{9}$ & $\mathbf{1 0}$ & $\mathbf{1 1}$ & $\mathbf{1 2}$ \\
\hline Dept 1 & 0 & 0 & 0 & 0 & 0 & 0 & 0 & 0 & 0 & 0 & 0 & 0 \\
\hline Dept 2 & 0 & 0 & 0 & 0 & 0 & 0 & 0 & 0 & 0 & 0 & 0 & 0 \\
\hline Dept 3 & 0 & 0 & 0 & 0 & 0 & 0 & 0 & 0 & 0 & 1 & 0 & 0 \\
\hline Dept 4 & 0 & 0 & 0 & 0 & 0 & 0 & 0 & 0 & 0 & 0 & 0 & 0 \\
\hline Dept 5 & 0 & 0 & 0 & 0 & 0 & 0 & 0 & 0 & 0 & 0 & 0 & 0 \\
\hline Dept 6 & 0 & 0 & 0 & 1 & 0 & 0 & 0 & 0 & 0 & 1 & 0 & 0 \\
\hline Dept 7 & 0 & 0 & 0 & 0 & 0 & 0 & 0 & 0 & 0 & 0 & 0 & 0 \\
\hline Dept 8 & 0 & 0 & 0 & 0 & 0 & 0 & 0 & 0 & 0 & 0 & 0 & 0 \\
\hline Dept 9 & 0 & 0 & 0 & 0 & 0 & 0 & 0 & 0 & 0 & 0 & 0 & 0 \\
\hline Dept 10 & 0 & 0 & 0 & 0 & 0 & 0 & 0 & 0 & 0 & 0 & 0 & 1 \\
\hline Dept 11 & 0 & 0 & 0 & 0 & 0 & 0 & 0 & 0 & 0 & 0 & 0 & 0 \\
\hline Dept 12 & 0 & 0 & 1 & 0 & 0 & 1 & 0 & 0 & 0 & 0 & 0 & 0 \\
\hline
\end{tabular}

Fuente: elaboración propia

Tabla 4. Tiempo de producción (PT)

\begin{tabular}{|l|c|c|c|c|c|c|c|c|c|c|c|c|}
\hline & \multicolumn{10}{|c|}{ Departamento } \\
\hline & $\mathbf{1}$ & $\mathbf{2}$ & $\mathbf{3}$ & $\mathbf{4}$ & $\mathbf{5}$ & $\mathbf{6}$ & $\mathbf{7}$ & $\mathbf{8}$ & $\mathbf{9}$ & $\mathbf{1 0}$ & $\mathbf{1 1}$ & $\mathbf{1 2}$ \\
\hline Producto 1 & 5 & 4 & 5 & 6 & 7 & 9 & 2 & 2 & 4 & 5 & 6 & 2 \\
\hline Producto 2 & 2 & 3 & 4 & 4 & 3 & 3 & 3 & 9 & 9 & 5 & 6 & 5 \\
\hline Producto 3 & 4 & 3 & 5 & 2 & 2 & 6 & 5 & 2 & 6 & 4 & 2 & 5 \\
\hline Producto 4 & 3 & 2 & 4 & 6 & 2 & 5 & 4 & 3 & 2 & 3 & 3 & 5 \\
\hline
\end{tabular}

Fuente: elaboración propia
Tabla 3.Turnos DPER

\begin{tabular}{|l|l|l|l|l|l|}
\hline & \multicolumn{5}{|c|}{ Día } \\
\hline & 1 & 2 & 3 & 4 & 5 \\
\hline Turno 1 & 1 & 0 & 0 & 0 & 0 \\
\hline Turno 2 & 1 & 0 & 0 & 0 & 0 \\
\hline Turno 3 & 1 & 0 & 0 & 0 & 0 \\
\hline Turno 4 & 0 & 1 & 0 & 0 & 0 \\
\hline Turno 5 & 0 & 1 & 0 & 0 & 0 \\
\hline Turno 6 & 0 & 1 & 0 & 0 & 0 \\
\hline Turno 7 & 0 & 0 & 1 & 0 & 0 \\
\hline Turno 8 & 0 & 0 & 1 & 0 & 0 \\
\hline Turno 9 & 0 & 0 & 1 & 0 & 0 \\
\hline Turno 10 & 0 & 0 & 0 & 1 & 0 \\
\hline Turno 11 & 0 & 0 & 0 & 1 & 0 \\
\hline Turno 12 & 0 & 0 & 0 & 1 & 0 \\
\hline Turno 13 & 0 & 0 & 0 & 0 & 1 \\
\hline Turno 14 & 0 & 0 & 0 & 0 & 1 \\
\hline Turno 15 & 0 & 0 & 0 & 0 & 1 \\
\hline
\end{tabular}

Fuente: elaboración propia
Tabla 5. Ruta de producción (PR)

\begin{tabular}{|l|c|c|c|c|c|c|c|c|c|c|c|c|}
\hline & \multicolumn{10}{|c|}{ Departamento } \\
\hline & $\mathbf{1}$ & $\mathbf{2}$ & $\mathbf{3}$ & $\mathbf{4}$ & $\mathbf{5}$ & $\mathbf{6}$ & $\mathbf{7}$ & $\mathbf{8}$ & $\mathbf{9}$ & $\mathbf{1 0}$ & $\mathbf{1 1}$ & $\mathbf{1 2}$ \\
\hline Producto 1 & 1 & 1 & 1 & 0 & 1 & 1 & 0 & 1 & 1 & 0 & 0 & 1 \\
\hline Producto 2 & 0 & 0 & 1 & 1 & 0 & 0 & 1 & 0 & 1 & 0 & 0 & 0 \\
\hline Producto 3 & 0 & 1 & 1 & 1 & 0 & 0 & 1 & 1 & 0 & 1 & 0 & 0 \\
\hline Producto 4 & 1 & 1 & 0 & 0 & 1 & 0 & 0 & 1 & 0 & 0 & 0 & 0 \\
\hline
\end{tabular}

Fuente: elaboración propia 
La figura 3 muestra la programación de los movimientos de los departamentos obtenida al utilizar el modelo propuesto en este artículo. En este caso, el departamento 10 era precedencia del departamento 12, por lo que el movimiento del departamento 12 no inició sino hasta el periodo 11, cuando el departamento 10 había finalizado la fase de transporte de su movimiento. Adicionalmente, las tablas 6 y 7 muestran la producción en cada turno y el inventario al final de cada día de cada producto obtenido.

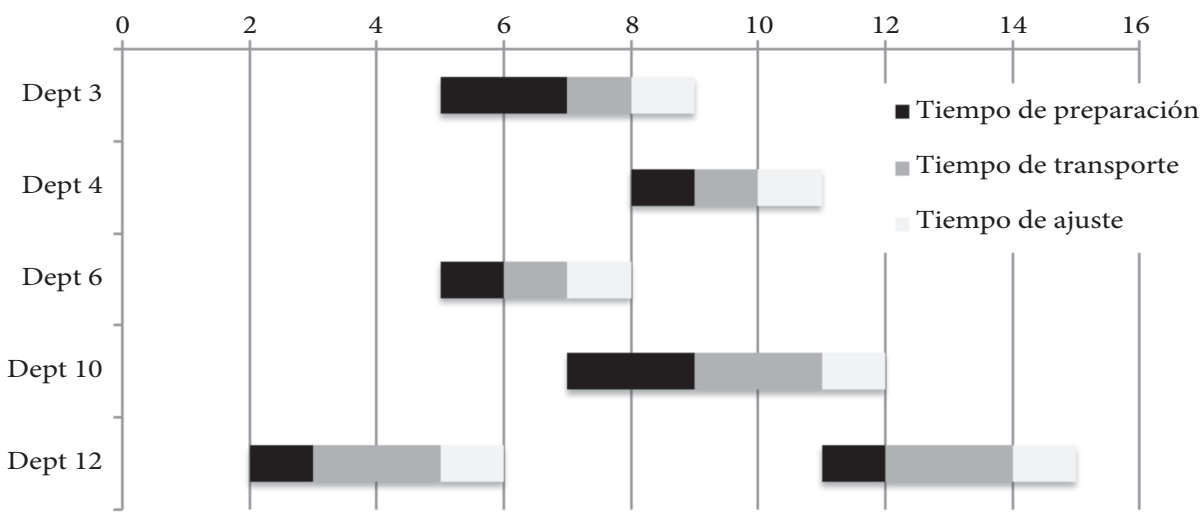

Figura 3. Programación de las actividades

Fuente: elaboración propia

Tabla 6. Producción

\begin{tabular}{|l|c|c|c|c|}
\hline & Prod 1 & Prod 2 & Prod 3 & Prod 4 \\
\hline Turno 1 & 51 & 4 & 41 & 0 \\
\hline Turno 2 & 0 & 0 & 0 & 10 \\
\hline Turno 3 & 0 & 53 & 0 & 0 \\
\hline Turno 4 & 0 & 0 & 6 & 0 \\
\hline Turno 5 & 0 & 0 & 0 & 34 \\
\hline Turno 6 & 0 & 0 & 0 & 0 \\
\hline Turno 7 & 0 & 0 & 0 & 0 \\
\hline Turno 8 & 0 & 0 & 0 & 0 \\
\hline Turno 9 & 0 & 0 & 0 & 0 \\
\hline Turno 10 & 0 & 0 & 0 & 0 \\
\hline Turno 11 & 0 & 0 & 0 & 0 \\
\hline Turno 12 & 0 & 0 & 0 & 0 \\
\hline Turno 13 & 0 & 0 & 0 & 14 \\
\hline Turno 14 & 0 & 0 & 0 & 0 \\
\hline Turno 15 & 13 & 8 & 10 & 0 \\
\hline
\end{tabular}

Fuente: elaboración propia

Tabla 7. Inventario

\begin{tabular}{|l|c|c|c|c|}
\hline & Prod 1 & Prod 2 & Prod 3 & Prod 4 \\
\hline Día 1 & 39 & 40 & 27 & 0 \\
\hline Día 2 & 24 & 29 & 23 & 25 \\
\hline Día 3 & 10 & 12 & 12 & 14 \\
\hline Día 4 & 0 & 0 & 0 & 0 \\
\hline Día 5 & 0 & 0 & 0 & 0 \\
\hline
\end{tabular}

Fuente: elaboración propia
La figura 4 presenta los consiguientes movimientos temporales.

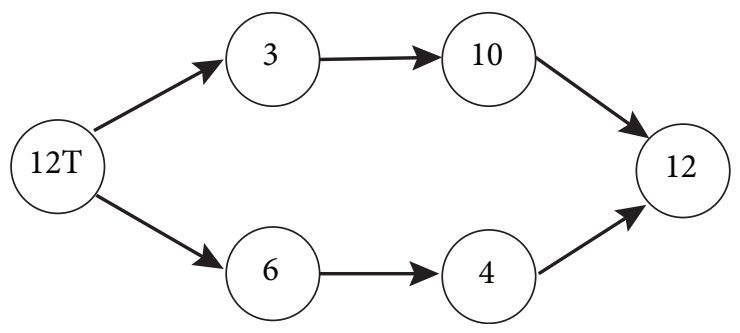

Figura 4. Grafo de precedencias final Fuente: elaboración propia

El proyecto tuvo una duración de 15 días, y el costo de traslado de los departamentos fue de $\$ 4800$. Para hallar este costo, no se tuvo en cuenta el costo de trasladar los departamentos no productivos.

\section{Resultados}

El modelo planteado por Lacksonen y Hung [3] no tiene en cuenta la planeación de la producción al momento de minimizar los costos de traslado de los departamentos y la duración del proyecto. Cuando la utilización de las máquinas de la planta es muy baja, no es necesario tener en cuenta la planeación de la producción durante el proyecto de redistribución, ya que las máquinas tienen capacidad 
de sobra para cumplir con la demanda. Sin embargo, cuando la utilización de las máquinas de la planta es alta, es necesario tener en cuenta la planeación de la producción durante el proyecto de redistribución para que la planta pueda cumplir con la demanda. En esta sección, se presenta la utilización de las máquinas de la planta donde es necesario tener en cuenta la planeación de la producción durante el proyecto de redistribución de planta.

Para encontrar esta utilización, se generaron 30 escenarios aleatorios como se muestran en la tabla 8, en los cuales el número de departamentos oscila entre 3 y 14 y se producen entre 3 y 13 productos. En cada uno de estos escenarios, se halló la utilización de las máquinas de la planta mínima que hace necesario tener en cuenta la planeación de la producción durante el proyecto de redistribución de planta.

Tabla 8. Resultados utilización de las máquinas de la planta

\begin{tabular}{|c|c|c|c|c|}
\hline Escenarios & $\begin{array}{c}\text { N. } \\
\text { Deptos }\end{array}$ & $\begin{array}{c}\mathrm{N} .^{\circ} \\
\text { Turnos }\end{array}$ & $\begin{array}{c}\mathrm{N} .^{\circ} \\
\text { Productos }\end{array}$ & Utilización \\
\hline 1 & 4 & 12 & 3 & $49 \%$ \\
\hline 2 & 4 & 19 & 3 & $43 \%$ \\
\hline 3 & 3 & 19 & 3 & $53 \%$ \\
\hline 4 & 4 & 19 & 3 & $50 \%$ \\
\hline 5 & 4 & 19 & 3 & $37 \%$ \\
\hline 6 & 4 & 19 & 3 & $62 \%$ \\
\hline 7 & 3 & 19 & 3 & $54 \%$ \\
\hline 8 & 4 & 19 & 3 & $56 \%$ \\
\hline 9 & 4 & 19 & 3 & $57 \%$ \\
\hline 10 & 3 & 19 & 3 & $65 \%$ \\
\hline 11 & 4 & 19 & 3 & $55 \%$ \\
\hline 12 & 4 & 19 & 3 & $37 \%$ \\
\hline 13 & 3 & 19 & 3 & $55 \%$ \\
\hline 14 & 4 & 19 & 3 & $41 \%$ \\
\hline 15 & 4 & 19 & 3 & $43 \%$ \\
\hline 16 & 3 & 19 & 3 & $50 \%$ \\
\hline 17 & 4 & 19 & 3 & $45 \%$ \\
\hline 18 & 4 & 19 & 3 & $66 \%$ \\
\hline 19 & 9 & 17 & 8 & $65 \%$ \\
\hline 20 & 9 & 17 & 8 & $67 \%$ \\
\hline 21 & 9 & 17 & 8 & $60 \%$ \\
\hline 22 & 9 & 17 & 8 & $54 \%$ \\
\hline 23 & 9 & 17 & 8 & $63 \%$ \\
\hline 24 & 9 & 17 & 8 & $69 \%$ \\
\hline 25 & 9 & 17 & 8 & $62 \%$ \\
\hline 26 & 9 & 17 & 8 & $64 \%$ \\
\hline 27 & 9 & 17 & 8 & $57 \%$ \\
\hline 28 & 9 & 17 & 8 & $59 \%$ \\
\hline 29 & 14 & 22 & 13 & $68 \%$ \\
\hline 30 & 14 & 22 & 13 & $60 \%$ \\
\hline
\end{tabular}

Fuente: elaboración propia
A partir de la tabla 8, se encontró la probabilidad que sea necesario considerar la planeación de la producción durante el proyecto de redistribución dada la utilización de las máquinas de la planta (figura 5). Por ejemplo, para una utilización del $10 \%$ hay una probabilidad del $0 \%$ de que sea necesario considerar la planeación de la producción, mientras que para una utilización del $60 \%$ hay una probabilidad del $63 \%$ de que sea necesario considerar la planeación de la producción.

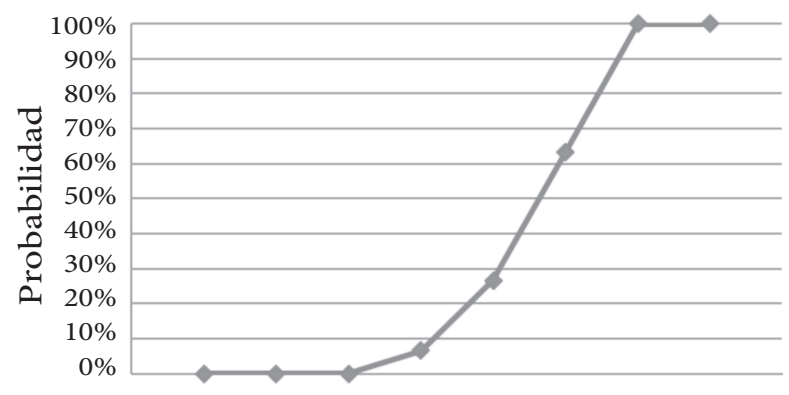

$\begin{array}{llllllllll}0 \% & 10 \% & 20 \% & 30 \% & 40 \% & 50 \% & 60 \% & 70 \% & 80 \% & 90 \%\end{array}$

Utilización de las máquinas de la planta

Figura 5. Probabilidad de considerar la planeación de la producción: análisis de la utilización de las máquinas de la planta Fuente: elaboración propia

A partir de los resultados obtenidos, se puede concluir que, para una utilización de las máquinas de la planta mayor al 60\%, es mejor utilizar este modelo, ya que al utilizar el modelo de Lacksonen y Hung [3], existe un $63,3 \%$ de probabilidad de que no sea posible cumplir con la demanda, lo que indica que es viable utilizar el modelo de Lacksonen y Hung [3] cuando en una instalación de manufactura tenga baja utilización de máquinas; específicamente para la utilización de las máquinas menores al 40\%, no es necesario considerar la planeación de la producción durante el proyecto de redistribución. Sin embargo, cuando la utilización de las máquinas es superior al $60 \%$, es mejor utilizar el modelo propuesto en este artículo, ya que garantiza el cumplimiento de la demanda. Cabe anotar que, en un entorno tan competitivo como el actual, las empresas están buscando siempre cómo maximizar el uso de los recursos, lo cual lleva a que en la práctica, en contextos como el colombiano, sea más común contar con utilizaciones de máquinas mayores al $60 \%$, resaltando la importancia del modelo propuesto en este artículo. 


\section{Conclusiones y campos futuros de investigación}

En este artículo, se plantea un modelo de programación lineal entera mixta para realizar la programación de las actividades en un proyecto de redistribución de planta conjuntamente con la planeación de la producción, de tal manera que se garantice el cumplimiento de la demanda durante el proyecto de redistribución de planta.

Este modelo es aplicable en empresas que tengan maquinaria liviana, en las cuales los costos asociados con el traslado y la instalación de los departamentos son bajos, de tal manera que estos pueden producir en una posición temporal. Adicionalmente, al garantizar el cumplimiento de la demanda, el modelo se vuelve más aplicable en la realidad, pues no cumplir con la demanda es una de las mayores preocupaciones de las empresas a la hora de realizar un proyecto de redistribución de planta [6].

Los resultados sugieren que empresas que tengan una utilización de las máquinas mayor al $60 \%$ deben utilizar el modelo propuesto en este artículo a la hora de realizar la programación de las actividades en un proyecto de redistribución de planta, pues al utilizar el modelo propuesto por [3], existe un $63,3 \%$ de probabilidades de no cumplir con la demanda.

En el futuro se puede incluir en el modelo la disminución de la eficiencia que existe cuando un departamento se encuentra produciendo en una posición temporal, ya que esto afecta directamente el cumplimiento de la demanda y, al tener en cuenta esta disminución, el resultado sería mucho más cercano a la realidad. Con este mismo propósito, se puede modificar el modelo para que reciba una demanda estocástica.

En muchos proyectos de redistribución de planta se incluye nueva maquinaria y estaciones de trabajo lo que implica una expansión o contracción de algunos departamentos. En tales casos, es importante que el modelo pueda considerar estos cambios. Adicionalmente, se puede incluir en el modelo un algoritmo que permita determinar las precedencias que son encontradas manualmente sobreponiendo la distribución final con la inicial.

En algunas industrias como la química o la médica, existen departamentos productivos que no pueden estar juntos, debido a esto es relevante agregar al modelo una matriz de adyacencias que indique cuáles departamentos pueden estar juntos en la posición temporal.

\section{Referencias}

[1] L. Nicol y R. Hollier, "Plant layout in practice", Material flow, vol. 1, pp. 101-107, 1985.

[2] S. Kulturel-Konak, A. E. Smith y B. A. Norman, "Biobjective facility expansion and relayout considering monuments", IIE Transactions, vol. 39, n. ${ }^{\circ}$ 7, pp. 747761, 2007.

[3] T. Lacksonen y C.-Y. Hung, "Project scheduling algorithms for re-layout projects", IIE Transactions, vol. 30, n. ${ }^{\circ} 1$, pp. 91-99, 1998.

[4] J. Driscoll y J. Sawyer, "A computer model for investigating the re-layout of batch production areas", International Journal of Production Research, vol. 23, n. ${ }^{\circ}$ 4, pp. 783-794, 1985.

[5] T. Lacksonen y M. Vijayvargiya, "Project scheduling for re-layout projects", Industrial Engineering Research Conference, pp. 566-570, 1994.

[6] L. Rivera, L. Cardona, L. Vásquez y M. Rodríguez, "Selección de alternativas de redistribucion de planta: Un enfoque desde las organizaciones", Revista S\& $T$, vol. 10, n. ${ }^{\circ} 23$, pp. 9-26, 2012.

[7] M. Rosenblatt, "The dynamics of plant layout", Managment Science, vol. 32, n. ${ }^{\circ}$ 1, pp. 76-86, 1986.

[8] R. Batta, "The dynamics of plant layout", Management Science, vol. 33, n. ${ }^{\circ}$ 8, pp. 10-65, 1987.

[9] Urban, "Solution procidures for the dynamic facility layout problem”, Ann. Opper, vol. 76, pp. 323-342, 1998.

[10] T. Urban, "Computacional performance and efficiency of lower-bound procidures for the dinamic facility layout problem", Eur. J. Opper, vol. 57, n. ${ }^{\circ}$ 2, pp. 271-279, 1992.

[11] J. Balakrishnan, "The dynamics of plant layout", Management Science, vol. 39, n. ${ }^{\circ}$ 5, pp. 654-655, 1993.

[12] W. Liu, "Tabu search heuristics for the dynamic facility layout problem", M.S. thesis, Morgaton, West Virginia University, 2005.

[13] B. Montreuil y U. Venkaatadri, "Strategic interpolative design of dynamic manufacturing systems layout", Managment Science, vol. 37, n. ${ }^{\circ}$ 6, pp. 682-694, 1991.

[14] B. Montreuil y A. Laforge, "Dynamic layout design giving a scenario tree of probable futures", Eur J Oper Res, vol. 63, n. ${ }^{\circ} 2$, pp. 271-286, 1992.

[15] T. A. Lacksonen y E. Emory Enscore Jr., "Quadratic assignment algorithms for the dynamic layout problems", Inter J Prod Res, vol. 31, n. ${ }^{\circ}$ 3, pp. 503-517, 1993.

[16] T. Lacksonen, "Static and dynamic layout problems with varying areas", $J$ of the Operational Res Society, vol. 45, n. ${ }^{\circ}$ 1, pp. 59-69, 1994. 
[17] J. Balakrishnan y C. Cheng, "Dynamic layout algorithms: a state of the art survey", Omega, vol. 26, n. ${ }^{\circ} 4$, pp. 507-521, 1998.

[18] M. Mazinani, M. Abedzadeh y N. Mohebali, "Dynamic facility layout problem based on flexible bay structure and solving by genetic algorithm", Int J Adv Manuf Technol, vol. 65, n. ${ }^{\circ}$ 5-8, pp. 929-943, 2013.

[19] J. Tompkins y J. White, Facilities Planning, New York, NY: Jhon Wisley, 1984.

[20] R. A. Millen, M. M. Solomon y P. Afentakist, “The impact of a single input/output device on layout considerations in flexible manufacturing systems", Inter Jourl of Prod Res, vol. 30, n. ${ }^{\circ}$ 1, pp. 89-93, 1992.

[21] K. Han, S. Bae y D. Jeong, "A Matrix-Based Approach to the Facility Re-Layout Problem", Inter J of Math Models and Methods in Applied Sciencies, vol. 7, n. ${ }^{\circ}$ 5, pp. 584591, 2013.

[22] P. Hicks y T. Lowan, "CRAFT-M for layout arrangement”, Indust Engineering, vol. 8, n. ${ }^{\circ}$ 5, pp. 30-35, 1976.
[23] E. Ferrari, A. Pareschi, A. Persona y A. Regattieri, "Plant Layout Computerised Design: Logistics and Relayout Program (LRP)", Inter J of Adv Manuf Technol, vol. 21, pp. 917-922, 2003.

[24] M. Savsar, "Flexible facility layout by simulation", Computers \& Industrial Engineering, vol. 20, n. ${ }^{\circ}$ 1, pp. 155$165,1991$.

[25] A. Baykasoglu, T. Dereli y I. Sabuncu, "An ant colony algorithm for solving budget constrained and unconstrained dynamic facility layout problems", Omega, vol. 34, n. ${ }^{\circ}$ 4, p. 385-396, 2006.

[26] S. Kulturel-Konak, "Approaches to uncertainties in facility layout problems: Perspectives at the beginning of the 21st Century", Journal of Intell Manuf, vol. 18, n. ${ }^{\circ}$, pp. 273-284, 2007.

[27] G. Meng, S. S. Heragu y H. Zijm, "Reconfigurable layout problem”, Int J of Prod Res, vol. 42, n. ${ }^{\circ} 22$, pp. 4709-4729, 2004. 